\title{
DNA Minor Groove Binding
}

National Cancer Institute

\section{Source}

National Cancer Institute. DNA Minor Groove Binding. NCI Thesaurus. Code C40494.

DNA Minor Groove Binding involves temporary non-covalent interaction through intermolecular physical forces of attraction by a protein or low molecular weight ligand with double-stranded DNA in the smaller (minor) groove formed by the two antiparallel chains of the double helical structure, in either a sequence dependent or independent fashion. 